\title{
Sorafenib suppresses the cell cycle and induces the apoptosis of hepatocellular carcinoma cell lines in serum-free media
}

\author{
MINORU TOMIZAWA ${ }^{1}$, FUMINOBU SHINOZAKI ${ }^{2}$, TAKAO SUGIYAMA ${ }^{3}$, \\ SHIGENORI YAMAMOTO ${ }^{4}$, MAKOTO SUEISHI $^{3}$ and TAKANOBU YOSHIDA ${ }^{5}$ \\ Departments of ${ }^{1}$ Gastroenterology, ${ }^{2}$ Radiology,${ }^{3}$ Rheumatology, ${ }^{4}$ Pediatrics, and ${ }^{5}$ Internal Medicine, \\ National Hospital Organization Shimoshizu Hospital, Yotsukaido, Chiba 284-0003, Japan
}

Received May 28, 2010; Accepted July 19, 2010

DOI: $10.3892 /$ etm.2010.131

\begin{abstract}
To suppress the invasion of hepatocellular carcinoma (HCC) cells into surrounding connective tissues during metastasis, we investigated the usefulness of sorafenib. In order to search for model cell lines, cell numbers were counted to reveal cell lines with the potential to proliferate in serum-free media. Cell proliferation and cell motility were analyzed with the MTS and wound assay, respectively. 5-Bromo-2'-deoxyuridine (BrdU) labeling and mitotic and apoptotic indices were analyzed to assess the cell cycle and apoptosis. The expression levels of cyclin D1 and the cleavage of caspase- 3 were analyzed by Western blotting. HLF cells exhibited growth in the serum-free medium, while the other cell lines examined did not. Sorafenib suppressed the cell proliferation and motility of the HLF cells in the serum-free media. Both indices of BrdU and mitotic potential decreased and the apoptotic index was increased in the serum-free media with sorafenib, suggesting that the cell cycle was suppressed and apoptosis was induced. The expression levels of cyclin D1 decreased and the cleavage of caspase- 3 was noted in the serum-free media with sorafenib. Sorafenib may be suitable for molecular therapy to suppress the metastasis of HCC.
\end{abstract}

\section{Introduction}

Long-term infection of hepatitis B or C virus causes hepatocellular carcinoma (HCC) in liver cirrhosis. During metastasis, HCC cells invade connective tissues consisting of collagen or elastic fiber, since HCC nodules are surrounded by fibrous septa even at an early stage $(1,2)$. Invading HCC cells survive with mitotic potential in connective tissue, which provides low nutrition and growth factors $(2,3)$. It was revealed that a

Correspondence to: Dr Minoru Tomizawa, Department of Gastroenterology, National Hospital Organization Shimoshizu Hospital, 934-5 Shikawatashi, Yotsukaido, Chiba 284-0003, Japan E-mail: nihminor-cib@umin.ac.jp

Key words: 5-bromo-2'-deoxyuridine, wound assay, terminal deoxynucleotidyl transferase-mediated nick end labeling assay, cyclin D1, caspase-3 subclone of Lewis lung carcinoma was resistant to apoptosis when compared to its parent (4). This same phenomenon may occur during the invasion of connective tissue by HCC cells. For experiments involving the cell invasion of connective tissue, serum-free medium was used as a model of its microenvironment (5).

Sorafenib is a multikinase inhibitor with high efficacy against tumors (6). It suppresses cell proliferation and induces apoptosis in HCC cell lines (7). Sorafenib has been proven to be effective for HCC and is used in clinical settings (8). When sorafenib suppresses the cell proliferation and motility of HCC in invading connective tissue, it is believed that it can suppress metastasis at an early stage. However, there are no reports involving the use of sorafenib to suppress proliferation and motility in a serum-free condition.

We investigated the possibility that sorafenib suppresses the proliferation and motility of HCC cells in serum-free media to search for a molecular therapy for suppressing the invasion of connective tissue by HCC.

\section{Materials and methods}

Cell culture. The HCC cell lines (HLE, HLF, PLC/PRF/5, Huh-7 and Hep3B) and the hepatoblastoma cell lines (Huh-6 and HepG2) were purchased from Riken Cell Bank (Tsukuba, Japan) and cultured in Dulbecco's minimum essential medium (DMEM) (Sigma, St. Louis, MO, USA) supplemented with 10\% fetal bovine serum (FBS) (Life Technologies Japan, Tokyo, Japan) in $5 \% \mathrm{CO}_{2}$ at $37^{\circ} \mathrm{C}$ in a humidified chamber. Cells $\left(10^{4}\right)$ were spread onto each well of 6-well plates to count cell numbers. For the wound assay and analysis of 5-bromo2'-deoxyuridine (BrdU) labeling, mitosis and apoptosis, cells were spread onto 4-well chambers (Becton Dickinson, Franklin Lakes, NJ, USA).

Cell proliferation assay. Cells were trypsinized, harvested and spread onto 96-well flat bottom plates (Asahi Techno Glass, Funabashi, Japan) at a density of 1,000 cells per well. Following $24 \mathrm{~h}$ of culture in DMEM with 10\% FBS, medium was exchanged with DMEM without FBS to quench the FBS effects. After $24 \mathrm{~h}$ of culture in DMEM without FBS, sorafenib (JS Research Trading e Kfm, Wedel, Germany) was added to the medium. Seventy-two hours later, the 
A

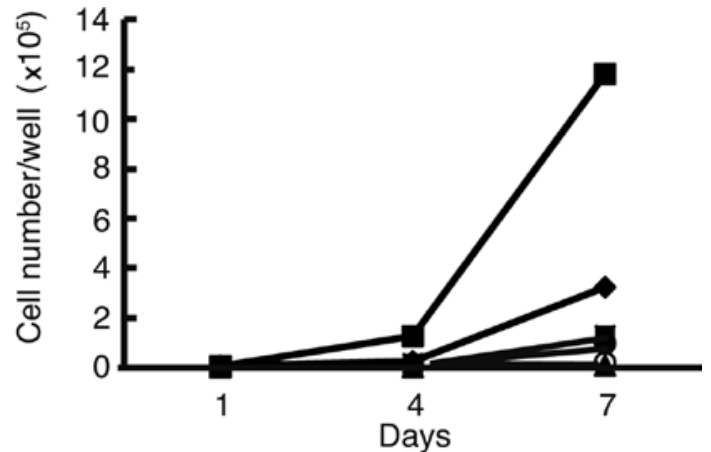

B

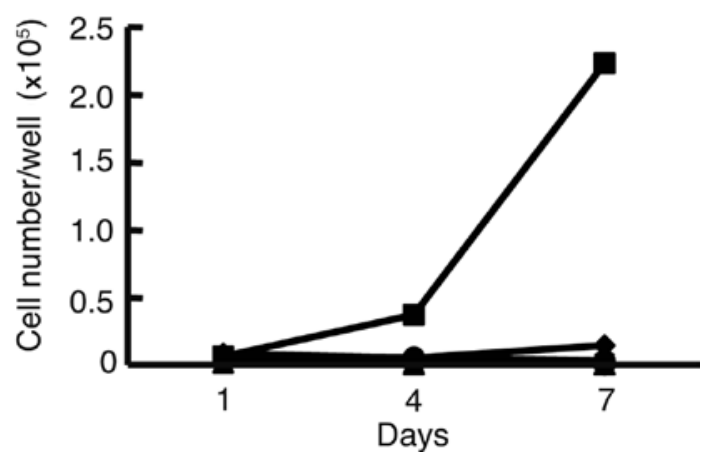

Figure 1. Growth curve of hepatocellular carcinoma and hepatoblastoma cell lines. Cell numbers were counted 1,4 and 7 days after the plating of $10^{4}$ cells onto each well of 6 -well plates in media with $10 \%$ FBS (A) or without FBS (B). HLE cells grew in medium with FBS, while HLF cells grew most rapidly in media without FBS. HLE (•), HLF (®), PLC/PRF/5 («), Huh-7 (x),

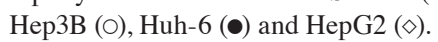

3-(4,5-dimethylthiazol-2-yl)-5-(3-carboxymethoxyphenyl)2-(4-sulfophenyl)-2H-tetrazolium inner salt (MTS) assay was performed according to the manufacturer's instructions (Promega Corporation, Tokyo, Japan). MTS was bio-reduced by the cells into a colored formazan product that reduces absorbance at $490 \mathrm{~nm}$. The absorbance was analyzed with a multiple plate reader at a wavelength of $490 \mathrm{~nm}$ with a Bio-Rad Model 550 microplate reader (Bio-Rad, Hercules, CA, USA). Raw data were normalized against those of $0 \mu \mathrm{M}$ sorafenib.

BrdU labeling, mitotic cells and apoptosis. Cells were incubated with BrdU at $10 \mu \mathrm{M}$ for $2 \mathrm{~h}$ and analyzed immunohistochemically according to the manufacturer's instructions (Roche, Tokyo, Japan). The numbers of cells positive for BrdU were counted for every 100 cells (BrdU labeling index). The numbers of cells during the mitotic phase were counted for every 100 cells using H\&E-stained slides (mitotic index). Apoptotic cells were detected by terminal deoxynucleotidyl transferase-mediated nick end labeling assay (TUNEL) staining, according to the manufacturer's instructions (Wako, Osaka, Japan). The numbers of cells positive for TUNEL were counted for every 100 cells (apoptotic index). For each index, the numbers of cells were counted for every 100 cells from five different fields.

Wound assay. A cut was made amid the cells with a sterile razor $24 \mathrm{~h}$ after plating onto 4 -well chamber and cells were stained with H\&E 72 h later (9). For each experiment, the number of HLF cells migrating $>150 \mu \mathrm{m}$ per $100 \mu \mathrm{m}$ cut surface was counted using microscopic images.
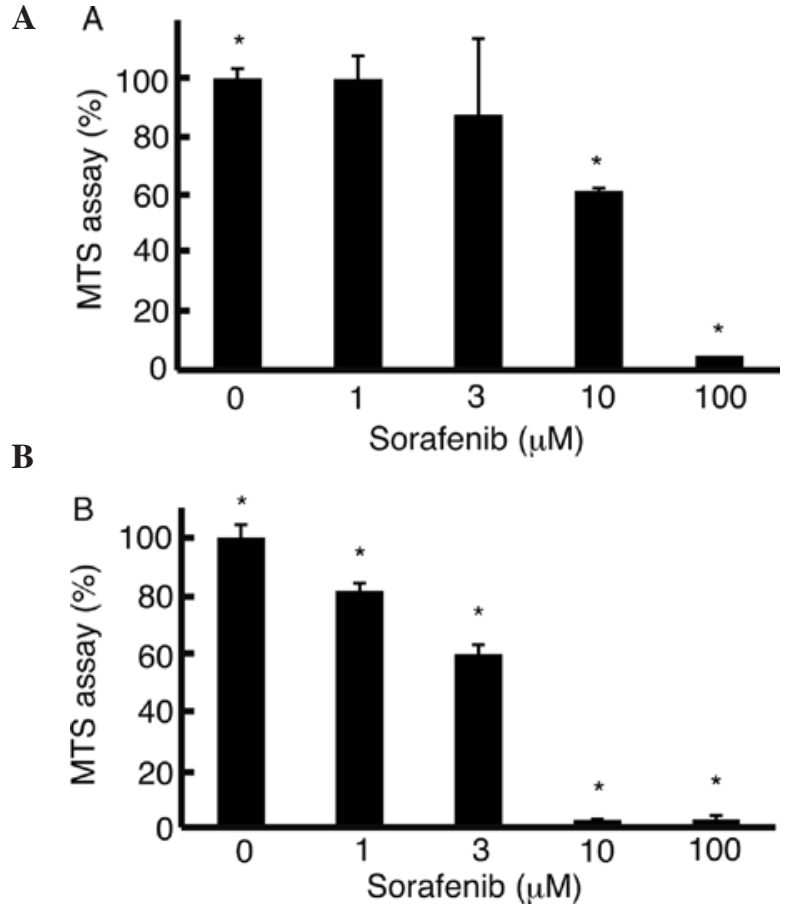

Figure 2. MTS assay. The MTS assay was performed to analyze the proliferation of HLF cells cultured in media with $10 \%$ FBS (A) and the proliferation of HLF cells in media without FBS (B) with sorafenib. Cell proliferation was suppressed as the concentration of sorafenib increased. In the media without FBS, cell proliferation was suppressed to a greater exent than the cell proliferation in the media with FBS, at the same concentrations of sorafenib. Error bar, standard deviation, ${ }^{*} \mathrm{P}<0.05, \mathrm{n}=3$.

Western blot analysis. Protein was isolated from the cells after $72 \mathrm{~h}$ of culture in a serum-free medium. Protein $(20 \mu \mathrm{g})$ was subjected to sodium dodecyl sulphate polyacylamide gel electrophoresis and transferred to a nylon filter. Primary antibodies were rabbit monoclonal anti-cyclin D1 antibody (Cell Signaling Technology, Danvers, MA, USA), rabbit monoclonal anti-caspase 3 antibody (Cell Signaling Technology) and mouse monoclonal anti-tubulin- $\alpha$ antibody (Lab Vision, Fremont, CA, USA). Secondary antibodies were the horseradish peroxidase (HRP)-linked anti-rabbit antibody and the HRP-linked anti-mouse antibody (both from GE Healthcare UK Ltd., Buckinghamshire, UK). Dilutions were 1:500 for the primary antibodies and 1:1,000 for the secondary antibodies. The filter was reprobed with anti-tubulin- $\alpha$ antibody. The specific antigen-antibody complexes were visualized by enhanced chemiluminescence (GE Healthcare UK Ltd.).

Statistical analysis. One-factor analysis of variance was performed with JMP5.0J (SAS Institute Japan, Tokyo, Japan). Values of $\mathrm{P}<0.05$ were accepted as statistically significant.

\section{Results}

We first analyzed the growth curve of all of the cell lines in media with or without FBS. In media with FBS, the cell numbers of all of the cell lines increased (Fig. 1A). The HLF cells exhibited rapid growth in the media without FBS when compared to growth in the media with FBS (Fig. 1B). Cell numbers of the other cell lines decreased compared to the 


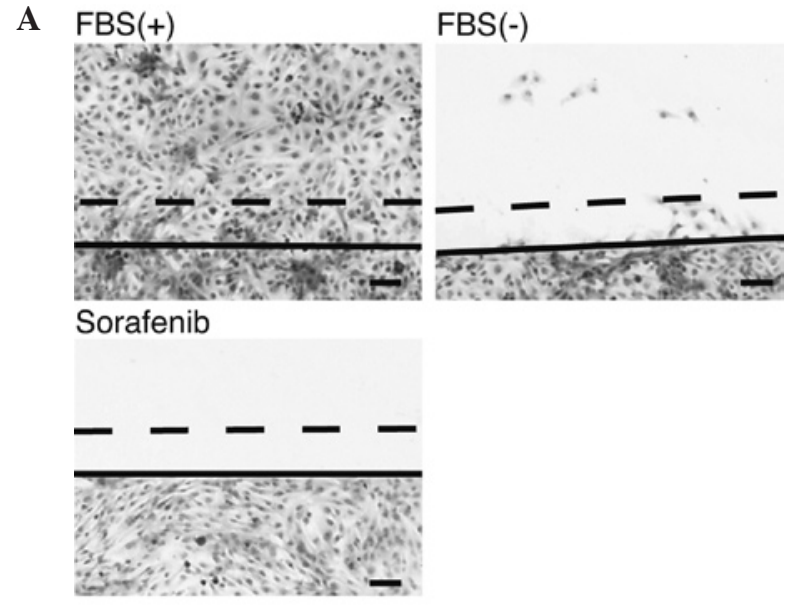

B

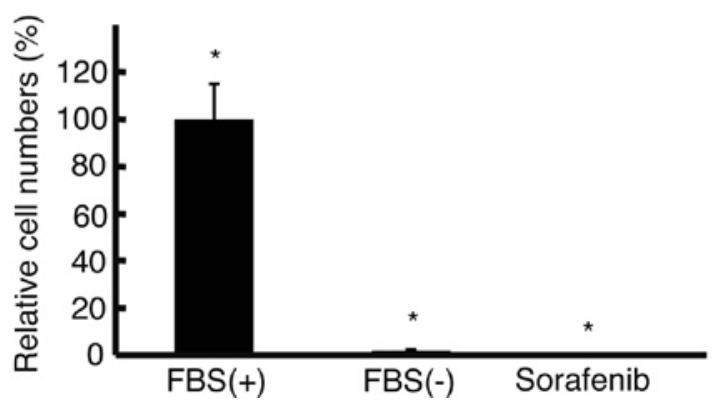

Figure 3. Wound assay. (A) The wound assay was performed to analyze cell motility using H\&E-stained culture slides. (B) The number of HLF cells migrating $>150 \mu \mathrm{m}$ per $100 \mu \mathrm{m}$ of cut surface was counted. No cells migrated $>150 \mu \mathrm{m}$ in the media without FBS with sorafenib (Sorafenib). Solid line in A, edge of scratch; dotted line in A, $150 \mu \mathrm{m}$ from the solid line. Original magnification, $\mathrm{x} 4$; scale bar, $100 \mu \mathrm{m}$. FBS(+), media with $10 \%$ FBS; FBS(-), media without FBS; Sorafenib, media without FBS with $10 \mu \mathrm{M}$ sorafenib.

HLE and HLF cells. Since the HLF cells grew most rapidly compared to the other cell lines examined in the media without FBS, HFL cells were used for further analysis.

To examine the suppression of cell proliferation by sorafenib, the MTS assay was performed. In the media with FBS, cell proliferation of the HLF cells was suppressed at $3 \mu \mathrm{M}$ sorafenib (Fig. 2A). In the media without FBS, suppression of cell proliferation occurred at $1 \mu \mathrm{M}$ sorafenib (Fig. 2B). At $10 \mu \mathrm{M}$ sorafenib, the MTS assay was $61 \pm 1 \%$ (mean \pm standard deviation) in the media with FBS, while it was $2.6 \pm 0.16 \%$ in the media without FBS $(\mathrm{P}<0.05)$, almost the same level as in the media with FBS at $100 \mu \mathrm{M}$ sorafenib $(2.7 \pm 1.3 \%)$ (Fig. 2B).

The wound assay was performed to analyze the effect of sorafenib on cell motility under a serum-free condition (Fig. 3A). In the media without FBS, $2 \pm 0.5 \%$ HLF cells migrated $>150 \mu \mathrm{m}$, which was significantly lower than that in the media with FBS $(\mathrm{P}<0.05)$ (Fig. 3B). No cells moved $>150 \mu \mathrm{m}$ in the serum-free media with sorafenib.

To clarify the effect of sorafenib on the cell cycle, the BrdU labeling and mitotic indices were analyzed. BrdU labeling index decreased in the media without FBS $(9.2 \pm 3.2 \%)$ as compared to the index in the media with FBS $(15.7 \pm 1.3 \%)(\mathrm{P}<0.05)$ (Fig. 4A). Upon the addition of sorafenib in the media without FBS,
$\mathbf{A}$

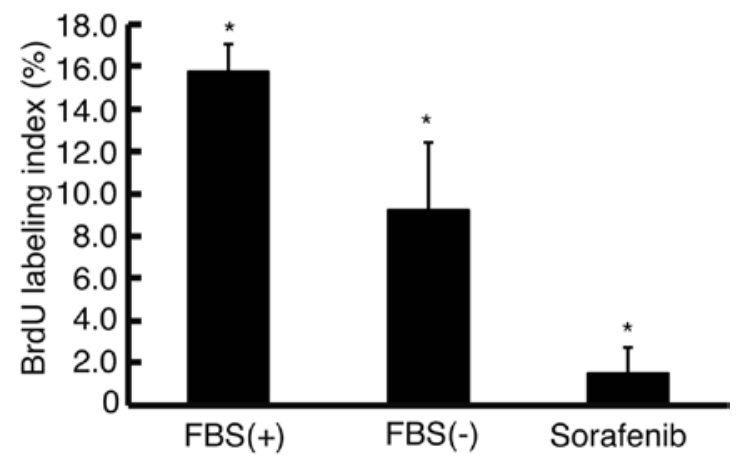

B

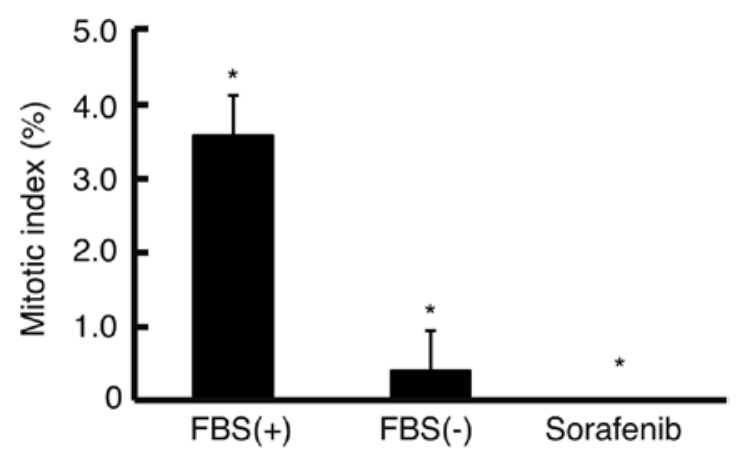

C

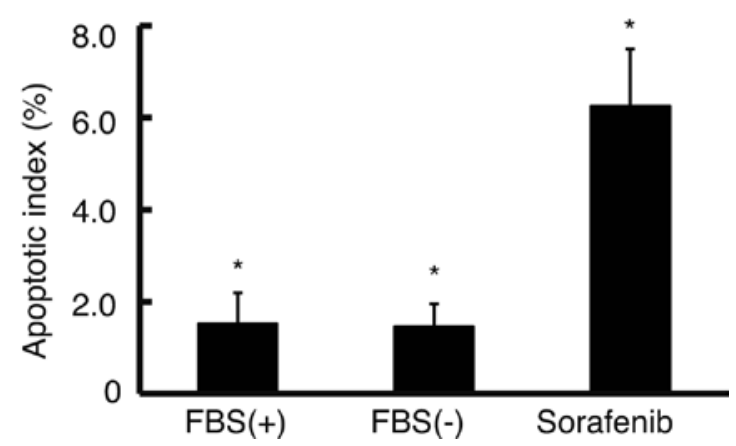

Figure 4. BrdU labeling, mitotic and apoptotic indices. (A) BrdU (10 $\mu \mathrm{M})$ was added to the culture medium, and the number of positive cells was counted to analyze DNA synthesis immunohistochemically. (B) Mitotic cell number was counted using H\&E-stained culture slides. (C) Apoptotic cell number was counted using culture slides with TUNEL assay. Sorafenib significantly decreased the numbers of both BrdU-positive and mitotic cells, while significantly increasing those of apoptotic cells in media without FBS (see text for the calculation of percentages). Error bar, standard deviation. ${ }^{*} \mathrm{P}<0.05, \mathrm{n}=3$. FBS(+), media with $10 \%$ FBS; FBS(-), media without FBS; Sorafenib, media without FBS with $10 \mu \mathrm{M}$ sorafenib.

the BrdU labeling index significantly decreased to $1.5 \pm 1.2 \%$ $(\mathrm{P}<0.05)$. The mitotic index decreased to $0.4 \pm 0.55 \%$ in the media without FBS, which was significantly lower than the index in the media with FBS $(3.6 \pm 0.55 \%)(\mathrm{P}<0.05)$ (Fig. 4B). Upon the addition of sorafenib in the media without FBS, the mitotic index was $0 \%$. To assess the apoptosis in the serumfree condition, the TUNEL assay was applied. The ratio of apoptotic cells did not differ between cells in the media with or without FBS (1.5 \pm 0.6 and $1.4 \pm 0.5 \%$, respectively) (Fig. 4C). Sorafenib significantly increased the ratio of apoptotic cells to $6.3 \pm 1.2 \%$ in the medium without FBS.

Western blot analysis (Fig. 5) was carried out to ascertain the mechanism of suppression of the DNA synthesis and stimulation of apoptosis by sorafenib. The expression of cyclin 


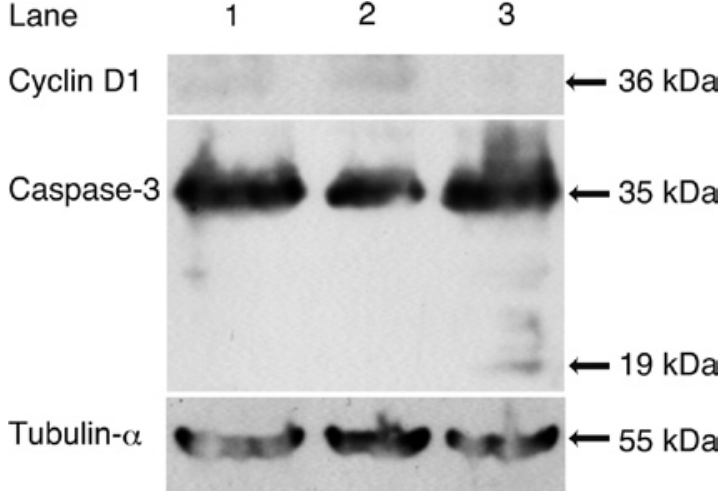

Figure 5. Western blot analysis. Western blot analysis was performed to analyze the molecular mechanism of the suppression of DNA synthesis and stimulation of apoptosis. The expression of cyclin D1 decreased (lane 2), while 19-kDa caspase-3, a cleaved form, was noted (lane 3 ) in cells in media with sorafenib. Lane 1: cells in media with $10 \%$ FBS; lane 2: cells in media without FBS; lane 3: cell in media without FBS with $10 \mu \mathrm{M}$ sorafenib.

D1 decreased in the cells in the media with sorafenib, while 19-kDa caspase-3, a cleaved form, was noted in the cells in the media with sorafenib.

\section{Discussion}

In the present study, HLF cells significantly exhibited more rapid proliferation in the media without FBS compared to the other cell lines examined, while HLE cells marginally exhibited proliferation. HLE and HLF cells are cloned from undifferentiated HCC and maintain the morphological features of poorly differentiated HCC such as a spindle shape (10). HLF cells exhibit the same shape in media with or without FBS, but their proliferation in a serum-free condition has not yet been analyzed (11). Our data clearly showed the significant proliferation of HLF cells in the media without FBS. Although the mechanism is unclear, HLF cells were found to maintain proliferation potential under a serumfree condition due to genetic alteration, such as p53 (11). Intriguingly, cells invading connective tissues surrounding HCC exhibit a spindle shape similar to HLF cells (2). In the present study, although the proliferative potential of the HLF cells in the media without FBS was weaker than that in the medium with FBS, as shown by the BrdU labeling and mitotic indices, they grew more rapidly than the other cell lines studied (Fig. 1B). The apoptotic index and the level of cleavage of caspase- 3 of the HLF cells in the media without FBS was the same as those in the media with FBS. HLF cells may survive under a serum-free condition not causing apoptosis for an unknown reason. A subclone of Lewis lung carcinoma was found to be resistant to apoptosis with a lower expression of caspase-3 (4). These data indicate that the surviving cells were resistant to apoptosis. HLF cells still marginally maintained the potential of motility. HLF cells were, thus, further analyzed as a model of the invastion of connective tissues by HCC cells.

In our study, $10 \mu \mathrm{M}$ of sorafenib suppressed cell viability to $61 \%$ in the media with FBS, which is higher than that in a previous report on PLC/PRF5 and HepG2 cells (7). This may be due to the fact that HLF cells have stronger proliferative potential than PLC/PRL/5 and HepG2 cells (Fig. 1A). Under a serum-free condition, suppression of cell viability was noted at $1 \mu \mathrm{M}$ of sorafenib and declined to $2.6 \%$ at $10 \mu \mathrm{M}$. These data suggest that a lower dose of sorafenib can suppress cell viability of HLF cells in a medium without FBS to a greater exent than that in medium with FBS. Sorafenib suppressed the cell cycle and stimulated apoptosis (Fig. 4). Sorafenib was also found to induce apoptosis in PLC/PRL/5 cells (7). Our data showed that sorafenib induced apoptosis in a serum-free condition. Notably, sorafenib completely abolished cell motility, as shown in the scratch assay (Fig. 3). These data indicate that sorafenib suppresses, not only cell proliferation, but also cell motility. Sorafenib significantly suppresses invasion of HCC cells to surrounding connective tissues.

\section{Acknowledgements}

This study was, in part, supported by a Grant-in-Aid for the Encouragement of Scientists from the Japan Society for the Promotion of Science (JSPS) (grant no. 22931047).

\section{References}

1. Kondo Y, Kondo F, Wada K and Okabayashi A: Pathologic features of small hepatocellular carcinoma. Acta Pathol Jpn 36: 1149-1161, 1986.

2. Tomizawa M, Kondo F and Kondo Y: Growth patterns and interstitial invasion of small hepatocellular carcinoma. Pathol Int 45: 352-358, 1995.

3. Gupta GP and Massague J: Cancer metastasis: building a framework. Cell 127: 679-695, 2006.

4. Takasu M, Tada Y, Wang JO, Tagawa M and Takenaga K: Resistance to apoptosis induced by microenvironmental stresses is correlated with metastatic potential in Lewis lung carcinoma. Clin Exp Metastasis 17: 409-416, 1999.

5. Boraldi F, Annovi G, Paolinelli-Devincenzi C, Tiozzo R and Quaglino D: The effect of serum withdrawal on the protein profile of quiescent human dermal fibroblasts in primary cell culture. Proteomics 8: 66-82, 2007.

6. Wilhelm SM, Carter C, Tang L, et al: BAY 43-9006 exhibits broad spectrum oral antitumor activity and targets the RAF/ $\mathrm{MEK} / \mathrm{ERK}$ pathway and receptor tyrosine kinases involved in tumor progression and angiogenesis. Cancer Res 64: 7099-7109, 2004.

7. Liu L, Cao Y, Chen C, et al: Sorafenib blocks the RAF/MEK/ ERK pathway, inhibits tumor angiogenesis, and induces tumor cell apoptosis in hepatocellular carcinoma model PLC/PRF/5. Cancer Res 66: 11851-11858, 2006.

8. Furuse J, Ishii H, Nakachi K, Suzuki E, Shimizu S and Nakajima K: Phase I study of sorafenib in Japanese patients with hepatocellular carcinoma. Cancer Sci 99: 159-165, 2008.

9. Pennisi PA, Barr V, Nunez NP, Stannard B and Le Roith D: Reduced expression of insulin-like growth factor I receptors in MCF-7 breast cancer cells leads to a more metastatic phenotype. Cancer Res 62: 6529-6537, 2002.

10. Dor I, Namba M and Sato J: Establishment and some biological characteristics of human hepatoma cell lines. Gann 66: 385-392, 1975.

11. Terai S, Noma T, Kimura T, Nakazawa A, Kurokawa F and Okita K: Wild-type p53 gene-induced morphological changes and growth suppression in hepatoma cells. J Gastroenterol 32: 330-337, 1997. 